\title{
Marketing sostenible y responsabilidad social organizacional: un camino hacia el desarrollo sostenible.
}

\section{Sustainable marketing and corporate social responsibility: a path towards sustainable development.}

\author{
Claudia Lorena Giraldo-Patiño ${ }^{1}$, José Londoño-Cardozo², Diana Carolina Micolta-Rivas ${ }^{3}$, \\ Estefanía O'neill-Marmolejo ${ }^{4}$ \\ ${ }^{1,3,4}$ Corporación Universitaria Minuto de Dios, Cúcuta - Colombia, ${ }^{2}$ Universidad Nacional de Colombia, Bogota - Colombia

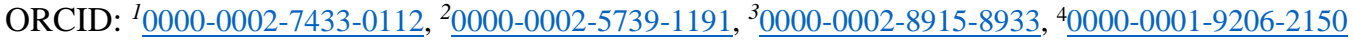

Recibido: 25 de octubre de 2020.

Aceptado: 19 de diciembre de 2020.

Publicado: 01 de enero de 2021.

\begin{abstract}
Resumen - Los impactos negativos al ambiente como la escasez de recursos naturales, el deterioro a los ecosistemas o los daños a la capa de ozono, se generan de manera rápida y creciente, debido al desarrollo industrial y el consumo indiscriminado de las personas como consecuencia del sistema económico dominante desde mediados del siglo XX. Esta situación impulsa una serie de prácticas que buscan solucionar dichas problemáticas y que se orientan a la conservación del entorno ambiental o a la solución de situaciones sociales. Entre estas aparece el marketing sostenible como una práctica integradora de los principios y materias fundamentales de la responsabilidad social organizacional (RSO). En tal sentido, este documento discute los principios del marketing sostenible y la RSO como un camino hacia la sostenibilidad que promueve un consumo y producción responsables. Por lo anterior, se plantean algunas estrategias de marketing encaminadas a responder los requerimientos de los consumidores, que se alinean con los objetivos organizacionales y los principios ambientales, a través del desarrollo de productos ecológicos. La metodología empleada es una revisión sistémica de literatura que parte de dos proyectos interinstitucionales que se relacionan en su preocupación por la sostenibilidad. El documento concluye que la aplicación de una Responsabilidad Social Fundamental es necesaria para que un correcto marketing sostenible pueda darse de forma correcta.

Palabras Clave: desarrollo sostenible, responsabilidad social empresarial, marketing ecológico, marketing verde, responsabilidad social corporativa, marketing social, marketing mix, estrategias de marketing sostenible.
\end{abstract}

\footnotetext{
Abstract - Negative impacts on the environment, such as the scarcity of natural resources, the deterioration of ecosystems or damage to the ozone layer, are generated rapidly and increasingly due to industrial development and indiscriminate consumption because of the dominant economic system since the middle 20th century. This encourages a series of practices that seek to solve these problems and that are oriented to the conservation of the environment or to the solution of social situations. Among these, sustainable Marketing appears as an integrating practice of the fundamental principles and matters of Corporate Social Responsibility. In this sense, this document discusses the principles of sustainable marketing and CSR as a path towards sustainability that promotes responsible consumption and production. Therefore, some marketing strategies are proposed to meet consumer requirements, which are aligned with organizational objectives and environmental principles, through the development of ecological products. The methodology used is a systemic literature review that starts from two inter-institutional projects that are related in their concern for sustainability. The document concludes that the application of a fundamental social responsibility is necessary for sustainable marketing to take place properly.
}

Keywords: Sustainable Development, Organizational Social Responsibility, Ecological Marketing, Green Marketing, Social Marketing, Marketing Mix, Sustainable Marketing strategies. 


\section{INTRODUCCIÓN}

Con la evolución industrial y el desarrollo tecnológico, se generó un creciente consumo que llegó a ser desmedido, y con este, políticas de producción propias del capitalismo. Ello, desencadenó el desarrollo de procesos productivos más acelerados que promovieron la aparición de prácticas poco amigables con el planeta [1]. Estas no solo han afectado el ecosistema natural, mediante practicas extractivas extremas, el gasto desmedido de recursos naturales o la despreocupación por las condiciones de las personas presentes y futuras, sino que originaron tendencias de mercado como la obsolescencia programada, el consumismo, la competencia desleal, entre muchas otras que corroen todos los aspectos de las relaciones organizacionales y personales [2]. Sin embargo, en la actualidad, prolifera la masificación de posturas que abogan por el cambio de dichas prácticas. La proclamación de los Objetivos de Desarrollo Sostenible [3], [4], la obligatoriedad de aplicaciones de políticas de responsabilidad social [5] y los cambios en las costumbres de consumo son un ejemplo de ello [6]. Todo esto ha obligado a que la academia y las organizaciones planteen nuevas posturas y prácticas, o que dirijan su mirada hacia planteamientos poco atendidos como el marketing sostenible.

Históricamente, el marketing ha sido el instrumento mediante el cual las organizaciones empresariales han comunicado sus estrategias y productos a los potenciales consumidores. Desafortunadamente, también se vio influenciado por los ideales del sistema hegemónico. De igual forma, se desarrollaron prácticas que hoy son discutidas. Desde mediados del siglo XX, se han propuesto posturas que rompen con esta hegemonía y procuran cumplir con los objetivos del marketing sin afectar ostensiblemente a la sociedad, al ambiente y en general al planeta.

Por otro lado, desde las organizaciones multilaterales, y gracias a la presión social, se promueve la adopción de políticas reales y efectivas de Responsabilidad Social Organizacional que contribuyan con la reducción de las externalidades de las operaciones, la dignificación de los puestos de trabajo y la protección de los recursos naturales para el disfrute de la sociedad actual y futura.

El marketing sostenible es la respuesta para el cambio de estos procedimientos, dado que integra en sus fundamentos los principios y las materias fundamentales de la Responsabilidad Social Organizacional. Ello permite la generación de actividades cuya masificación podría formar un nuevo camino hacia la sostenibilidad. Con este proceso, no solo se promueven acciones amigables con el ambiente, las buenas prácticas en la contratación, producción y uso de materias primas, sino que se invita a los consumidores a adoptar un consumo responsable. Todo ello, finalmente se ve reflejado en el cumplimiento de los objetivos organizacionales y en el posicionamiento de la organización como promotora de la sostenibilidad. Algo que finalmente generará un valor que le ubicará como una opción de preferencia para los clientes. Es decir, una externalidad positiva.

Dicho lo anterior, este documento presenta las bases conceptuales que unen al marketing con la Responsabilidad Social Organizacional y discute alrededor de los soportes teóricos del marketing sostenible. Este término se propone como una categoría que subsume a otros ampliamente utilizados como marketing social, marketing verde, marketing ecológico, entre otros.

Los términos de marketing dirigidos a la sostenibilidad y Responsabilidad Social emergieron como propuestas que se enfocaban hacia la solución de la problemática más evidente de cada momento [7]. Se considera que estas propuestas podían ser una evolución constante que incluía el interés en nuevas temáticas sostenibles. Sin embargo, distintos autores tienden a tratarlos como conceptos independientes [7]-[9].

El desarrollo teórico actualidentifica un punto común entre las propuestas. Para ello, es necesario contrastar cada uno de sus postulados con la teoría de la Responsabilidad Social Organizacional. El marketing sostenible agrupa a todas las demás propuestas semánticas, en cuanto cada una de ellas se enfoca en algunos de los principios y materias de la responsabilidad social; el marketing sostenible los tiene en cuenta todos.

Para todo lo anterior, el documento se ordena como sigue. En primer lugar, se presenta la fundamentación teórica. Aquí se presentan las discusiones acerca de la Responsabilidad Social Organizacional, sus diferencias semánticas y el enfoque que se acoge. También, se abordan los fundamentos de marketing y se discuten los términos con enfoque sostenible. En segundo lugar, se describe la metodología. En tercer lugar, se discute que integra el fundamento teórico de la responsabilidad social organizacional con el del marketing sostenible para sensibilizar su necesidad. En quinto lugar, se plantean algunas de las estrategias que se pueden trazar desde posturas de sostenibilidad en el marketing mix, y, en sexto lugar, se presentan las conclusiones.

\section{FUNDAMENTACIÓN TEÓRICA}

\section{a. Marketing}

El intercambio de productos y servicios es clave para las dinámicas sociales [10], [11]. En un principio, esto se basaba en el trueque, o cambio de un producto o servicio por otro al que se le asigna un valor similar. Es así como evoluciona el marketing, parte del intercambio y va hasta la compra y venta de los bienes tangibles o intangibles [12], [13].

El progreso del marketing ha sido continuo. Inicialmente su enfoque estuvo en producto, luego pasó al proceso, siguió con las ventas, posteriormente pasó al consumidor, para llegar a preocuparse por la competitividad [1], [11], [14]. Por lo anterior, la definición de marketing varia de acuerdo con la percepción, la aplicación y la finalidad que se tenga. En este documento se definirán brevemente algunos aspectos de sus fundamentos generales.

El marketing es un proceso social, mediante el cual las organizaciones e individuos logran alcanzar lo que necesitan o desean, a partir del intercambio de productos o servicios y la creación de relaciones redituables [13]. En estas, se atrae al cliente, mediante la oferta de bienes tangibles e intangibles que satisfacen sus necesidades, a la vez que se genera un beneficio económico para la organización [13], [15]. Otra definición por mencionar gira entorno a la visión de marketing como el conjunto de actividades realizadas que ayudan a que a la entidad consiga las metas propuestas mientras se anticipa a los deseos de los consumidores en el desarrollo de los productos o servicios requeridos [16]. Todo ello, por medio de procesos de comunicación, creación y entrega de valor, a través de actividades de intercambio entre la organización y un grupo de personas [17]. 
Las definiciones de marketing se relacionan con la segmentación, focalización y posicionamiento en el mercado, el desarrollo del branding y las estrategias del marketing mix [18]. Para ello, los principales autores del marketing definieron cuatro aspectos claves para su gestión. Se trata de las cuatro pes o 4 P. Este concepto hace referencia al desarrollo de estrategias de Precio, Plaza, Producto y Promoción [13], [19] (Figura 1). Con estas se busca identificar las necesidades de los clientes, establecer un precio óptimo al producto, caracterizar la plaza, la cadena de valor y la comunicación con los clientes y competidores del producto o servicio [13], [20], [21]. A continuación, se explicarán los factores del marketing mix.

Por producto se hace referencia a ese bien tangible o intangible que se pone a disposición de los clientes por parte de la organización. Las características de este producto son variadas. En esta $\mathrm{P}$ hay que tener en cuenta si trata de un bien o de un servicio, las funciones que desarrolla, la forma de uso o de interacción entre este y el cliente, sus características físicas y la diferenciación de este con otros bienes similares [13].

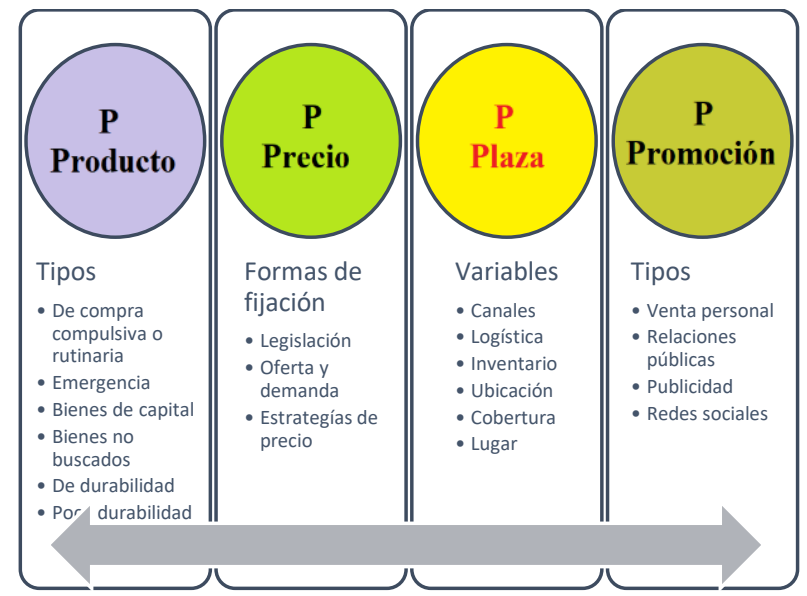

Figura 1: Las cuatro P del marketing

Fuente: Elaboración propia con base en aportes de [13], [19].

Se considera que el precio es uno de los factores a los que se encuentra ligada la supervivencia de las organizaciones [21]. Este es el rubro que será cobrado a los clientes por el bien o el servicio y para su fijación, se deben tener en cuenta algunos aspectos [13] (Figura 1).

La siguiente $\mathrm{P}$ corresponde a la plaza. En español esta $\mathrm{P}$ tiene diferentes acepciones que configuran el problema de sinonimia y polisemia de la administración descrito por Ríos-Szalay [22]. En el idioma inglés, corresponde al vocablo placement. Sin embargo, a nivel conceptual la claridad de este vocablo sobrepasa las traducciones al español.

Plaza hace referencia, específicamente, al medio mediante en el cual el cliente llega a los productos o servicios ofrecidos. La figura 1, expone las distintas variables en las que se puede interpretar esta P [13], [15], [21]. La última de las P corresponde a promoción. Esta se considera la base de lo que se conoce como mix marketing, o mezcla de estrategias de marketing, en español [16]. La promoción se encarga de recordar a los clientes la existencia de un producto o un servicio. El propósito principal de la promoción es influir en el consumidor para que opte por aquello que se le ofrece. En este sentido, es importante saber cuándo, cómo y dónde es posible transmitir los mensajes acerca del producto para que sean efectivos. Adicionalmente, en esta P se deciden los canales por los cuales este mensaje se hace llegar a su destinatario. Por lo general, se trata de medios tradicionales y no convencionales de comunicación [13].

\section{b. Responsabilidad social organizacional}

En este apartado se desarrollan los fundamentos de la responsabilidad social organizacional, en adelante RSO. Para ello se abordarán las diferencias semánticas alrededor del concepto a partir del trabajo de Tello-Castrillón y Rodríguez-Córdoba [23], [24]. Posteriormente, se presentan los fundamentos teóricos. En este punto, se desarrollan los principios fundamentales y las materias que se subsumen en la RSO a partir del trabajo de Tello-Castrillón y Londoño-Cardozo [25]. Todo esto para identificar a la RSO como un componente transversal a todos los asuntos de la organización; el marketing no es ajeno a ello.

La RSO enfoca su interés en el marketing cuando procura que se ejecuten actividades de marketing transparente con el consumidor, se haga una producción sostenible, se promueva el consumo responsable y sostenible y la reutilización. La RSO debe ser, por lo tanto, uno de los componentes fundamentales de la estrategia de toda organización. En términos de Tello-Castrillón [26], las estrategias de marketing deberían hacer parte de una política de RSO fundamental.

\section{1) Diferencias semánticas}

En el contexto académico existen diversos términos para hacer referencia a la responsabilidad social de las organizaciones. En español, el de mayor reconocimiento es Responsabilidad Social Empresarial (RSE) [23]. En el contexto anglosajón, el término de mayor institucionalización es Responsabilidad Social Corporativa (CSR, por sus siglas en inglés). Sin embargo, los nuevos modelos de responsabilidad social trascienden los escenarios netamente empresariales y se suscriben a todo tipo de organizaciones [23]-[25].

Desde la teoría organizacional las organizaciones se entienden como entes sociales, creadas por las personas [27], que se presentan como actores indiscutibles del acontecer social [28]. Ello, en tanto ofrecen una base de acción y de comportamiento a las personas [29]-[32], que fungen como escenario para el encuentro de los intereses y objetivos en común de los grupos e individuos de la sociedad [33]. Las organizaciones se pueden clasificar de distintas formas. Sin embargo, a grandes rasgos, hay una categorización que permite agrupar a todos sus tipos. Se trata de su ánimo u objetivo misional [34]. Sin bien, esta sistematización no es exacta en términos taxonómicos, se emplea para diferencias dos 
grandes clases [34]. Las empresas u organizaciones con ánimo de lucro son solo una de las subcategorías de las organizaciones si se toma este criterio clasificatorio [34].

Por lo anterior, se considera que el concepto que acoge las políticas de Responsabilidad Social y que se aplica a todos los tipos de organizaciones es el de Responsabilidad Social Organizacional, en adelante RSO [23], [25], [35]. Este es un término ampliamente discutido en la academia y que gana aceptación entre los expertos internacionales [36], [37]. De igual forma, las diferencias de la RSO frente a otros términos se fundamentan en la forma en cómo se concibe su aplicación.

\section{2) Fundamentos}

Algunos autores, especialmente quienes defienden conceptualmente el uso de RSE o CSR, consideraron que las acciones de responsabilidad social están direccionadas hacia la creación de valor para los accionistas [23], [25]. El autor de mayor reconocimiento en esta postura es Milton Friedman. Para el autor, "la responsabilidad de la empresa es aumentar las ganancias" [38, p. 1]. Sin embargo, posteriormente, otros autores relacionaron la responsabilidad social con el desarrollo sostenible [23], [25], [26].

Aßländer [39], Enderle [40] y Ungericht y Hirt [41] consideraron que la RSO está limitada a la moral intrínseca en la ética organizacional y para ello se debe procurar un equilibrio entre sus necesidades y las necesidades sociales. Para lograr ello, se debe establecer un código de conducta, o políticas de sustentabilidad [42]. Lo anterior identifica la RSO como una acción que debe ser transversal a todas las actividades de la organización [23], [25], [43], llevarse a cabo de forma voluntaria [23], [24], [43] y tener en cuenta a los grupos de interés [23], [25], [44][47]. A partir de lo anterior, Tello-Castrillón propuso que la responsabilidad social se puede entender de dos maneras. La RSO fundamental y la RSO instrumental o mecánica [26].

La primera de ellas es considerada como la verdadera RSO donde la organización se reconoce como un actor social o un vehículo meso societal donde se agrupan personas para alcanzar objetivos que no se pueden conseguir de forma individual. Las organizaciones que reconocen esto, independientemente de su fin, implementan las políticas de RSO de forma acertada [26]. En este sentido, Jenkins consideró que la verdadera RSO se presenta previo a la ganancia [43]. Esto significa que la organización no descarga sus costos, sociales, políticos, ambientales, entre otros, en la sociedad, sino que los asume en su modelo de negocio.

La RSO mecánica se presenta en las organizaciones que no cargan sus externalidades o costos en sus estados financieros [26]. El procedimiento que emplean, por lo general, consiste en mirar sus estados financieros finales e identificar si a partir de sus utilidades netas, es posible aplicar algunas acciones de RSO, comúnmente, con carácter altruista. Con esto, se desconoce la transversalidad de la RSO que empieza por el pago de salarios justos, el cumplimiento de la norma, el ofrecimiento de productos de calidad o sin obsolescencia programada, entre otras [26].

Por lo general, las organizaciones que emplean políticas de RSO mecánica se aprovechan de las rebajas de impuestos y subsidios que ofrece el Estado a cambio de financiación de obras, apertura de plantas de producción en zonas de bajos niveles de empleo y rebajas de impuestos, entre otros [26]. Entonces, la RSO mecánica está vinculada a las mejoras técnicas que las organizaciones implementan para favorecerse de las rebajas de impuestos, subsidios del Estado o como una herramienta de mercadeo [25], [26], [48].

\section{3) Principios}

A partir de los lineamientos de la norma ISO 26000 en versión 2010, se identifican los principios básicos de la RSO aquí propuesta [25] (figura 2). Estos deben ser aplicados por todas las organizaciones de forma transversal a sus actividades y se deben incorporar a la estrategia, es decir, deben integrarse como parte de una RSO fundamental.

No es necesaria la certificación en normas ISO para procurar cumplir los principios. De igual forma, también se considera que estos principios pueden ser aplicados por las personas en su cotidianidad. La interacción interpersonal que conecta a dos personas, como la economía colaborativa, debe estar sujeta a estos principios [49].

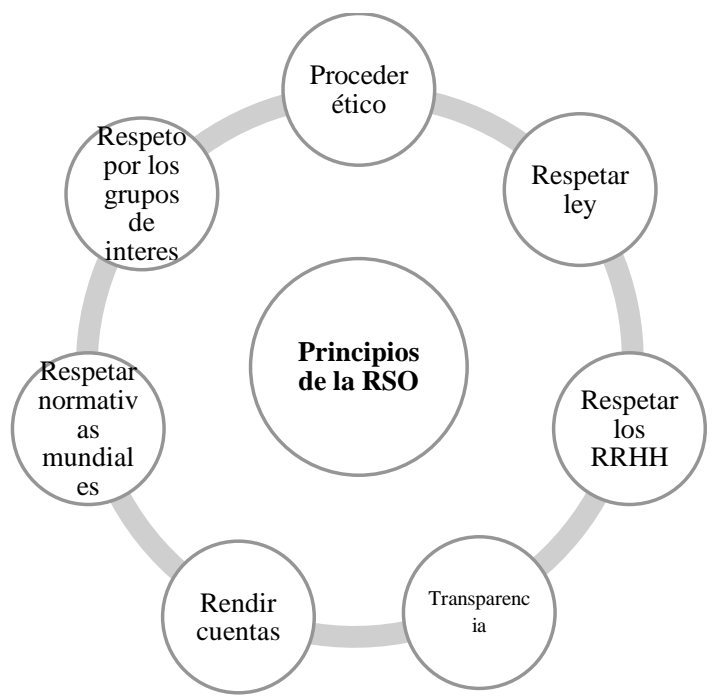

Figura 1: Principios de la responsabilidad social organizacional Fuente: Elaboración propia con base en aportes de [50] y [25]. 


\section{4) Materias}

Las materias de aplicación de la RSO son aquellos aspectos organizacionales donde se deben implementar con mayor medida los principios de la RSO [25]. En general, estas materias guardan relación con las áreas funcionales de las organizaciones. La primera materia por abordar es la gobernanza o gobierno corporativo [48], [51], [52].

El papel de la alta dirección de la entidad en la creación e implementación de las políticas de RSO es determinante. La gobernanza es uno de los principales temas de estudio acerca de la responsabilidad social [48], [52]. La gobernanza es una de las cualidades que debe tener el gobierno corporativo. Un gobierno corporativo justo, inclusivo y acorde con los lineamientos éticos es clave para que toda la organización asuma una actitud socialmente responsable [53], [54].

La segunda materia por mencionar está relacionada con las prácticas laborales. En esta se agrupa todo lo relacionado con el trabajode las personas a nivel interno y externo de la organización [25]. Este es uno de los aspectos que suelen dejarse de lado en las políticas de RSO. La tercera materia corresponde a las prácticas justas de operación [25] y se encuentra relacionada con las prácticas laborales y la relación con los grupos de interés externos. Está direccionada hacia evitar la corrupción, el soborno o la competencia desleal [4]. En seguida se encuentra la materia que tiene relación con el medio ambiente [25]; un tema en boga actualmente, dada su relevancia para la sostenibilidad del planeta. En tal sentido, las acciones de la organización deben retribuir positivamente su impacto ambiental.

La siguiente materia es la participación en el desarrollo activo de la comunidad [25]. Esta enfatiza en la retribución a la sociedad donde se desempeñan las actividades. Para ello, se identifica la cultura y las características socioculturales con la intención de influir en la colectividad mientras se garantiza el respeto de la identidad social [53], [54]. Finalmente, aparecen los asuntos de los consumidores [25] y las actividades o acciones digitales [55]. Para la primera, se pretende un interés por las acciones de los consumidores, se aboga por una verdadera estrategia de marketing que promueva el consumo responsable y sostenible, bienes y servicios utilizables por todos y que no estén en contra de los principios o de las otras materias de la RSO [25], [50]. Para el caso de las actividades digitales, es un nuevo material en el que se deben interesar las políticas de RSO dado el auge del uso de las tecnologías digitales por parte de personas y organizaciones [55]. Según Londoño-Cardozo, se debe realizar una adaptación de los principios y las materias de la RSO tradicional para subsumir las actividades organizacionales y personales en el contexto digital [55]. El autor le llama a esto responsabilidad digital organizacional o RDO [55].

\section{c. Inclinaciones del marketing hacia la sostenibilidad}

La evolución del marketing le ha hecho tomar distintos rumbos. Ello ocasiona el surgimiento diferentes categorías sobre las que se puede clasificar su aplicación [17], [56]. Sin embargo, las posturas hegemónicas del sistema capitalista permitieron una tendencia de consumo excesiva y poco sostenible [1], [11], [14]. En tal sentido, el marketing se ha especializado en lograr que las personas adquieran bienes y servicios que quizá no necesitaban o que a largo plazo no son amigables con el ambiente.

Adicionalmente, se han institucionalizado otras acciones como la obsolescencia programada o la oferta de bienes que no cumplen con las expectativas del cliente. Por lo cual, el marketing ha sido controversial en términos de la aplicabilidad que se le ha dado.

Desde la década de los 60 del siglo XX, se generó una creciente preocupación de las personas por los temas ambientales y sociales [56], [57]. A este respecto, emergen categorías de marketing que, impregnadas de las tendencias de RSO y sostenibilidad, buscan la corrección de las prácticas tradicionalmente institucionalizadas a partir del sistema económico hegemónico.

Tal es el caso de marketing ecológico, marketing verde, marketing social, marketing de perspectiva social, marketing sostenible y marketing de sostenibilidad [58]. Sin embargo, en la literatura académica no existe un consenso acerca de cuál término debe emplearse, ya que cada postura o tipo de marketing es diferente y tiene una finalidad distinta. Todos guardan en común su inclinación hacia el cuidado del medio ambiente [7]. Es por ello, que a continuación se presentará una breve revisión conceptual frente al tema.

Marketing ecológico es una corriente del marketing que surge desde la década de los setenta, debido a los accidentes en plantas productivas, que generaron contaminación ambiental significativa [7], [8], [59], [60]. Por ende, esta tipología se preocupa por los aspectos ambientales, en términos de la generación de impactos por el desarrollo de las actividades organizacionales. Principalmente fue empleado por ciertos sectores que generaban gran impacto ambiental negativo como las petroleras o empresas nucleares, especialmente por la obligatoriedad del desarrollo de normativas ambientales [7], [9], [61], [62].

El marketing verde o green marketing se orienta a satisfacer las necesidades de los clientes asociados a los impactos negativos generados por el producto [17]. Se estima que su surgimiento se da a partir de finales de los 80 del siglo XX [7]. Es así como esta categoría se centra en la disminución del impacto ambiental, a través del desarrollo de productos [63]. El marketing verde empezó a ser utilizado por organizaciones de diferentes sectores. No solo se enfocó en el proceso productivo, como el marketing ecológico, sino que también abordó al producto y su importancia como factor generador de residuos dadas las tendencias de consumo desmesurado [62]. En definitiva, esta categoría responde tanto a las preocupaciones sobre la contaminación ambiental como a las necesidades de los consumidores [62]-[66], [56].

La categoría de marketing social responde a las problemáticas sociales y se enfoca en influenciar el cambio en el consumidor. Por lo general, el principal objetivo de esta categoría es generar beneficios sociales, por lo cual es un tipo comúnmente usado por entidades sin ánimo de lucro [67], [68]. Por otro lado, la categoría de marketing de perspectiva social vincula el desarrollo del marketing a partir de los objetivos organizacionales y su alineación con los objetivos sociales. De esta manera, se desarrolla un marketing pensado en el desarrollo de productos que satisfagan las necesidades inmediatas a corto plazo, se generen beneficios sociales a largo plazo y se alcance así el éxito de la organización [58], [62]. La diferencia del primero sobre el segundo radica en su objetivo principal. El primero busca influir en el comportamiento del consumidor al generar conciencia sobre las consecuencias de prácticas abusivas del consumo de productos. El segundo, busca satisfacer las necesidades inmediatas del cliente, al responder a las preocupaciones sociales de corto y largo plazo mientras genera valor económico sostenible para la organización. 


\section{d. Marketing sostenible}

Las categorías expuestas anteriormente guardan cierta relación: el interés de las personas por las problemáticas sociales y ambientales que emergieron desde hace varias décadas. En efecto, este es un tema que se ha masificado en las últimas décadas. El uso indiscriminado de estos términos en la literatura genera confusión y tergiversa los conceptos respecto a sus enfoques y alcances. La relación entre las organizaciones empresariales y el medio ambiente se puede rastrear hasta, por lo menos, comienzos del siglo XX [7]. A pesar de ello, es posible identificar que hay una categoría, o, en términos de filosofía de la ciencia, una supracategoría [69]-[71] que subsume a todas las anteriores. Se trata del marketing sostenible.

En el marco del desarrollo sostenible, las organizaciones están llamadas a realizar acciones que integren tres ámbitos para generar un auténtico impacto [72]. Se trata de lo social, lo ambiental y lo económico. Con estos, se busca satisfacer las necesidades presentes de la sociedad actual, sin afectar la satisfacción de las necesidades futuras [57]. En términos de RSO a esto se le conoce como la triple línea de base o triple bottom line [53], [54], [73]. De esta manera, se busca la adopción de medidas viables económicamente que lleven a minimizar el deterioro ambiental y respondan a las preocupaciones sociales $[61]$ mientras se aplican las materias y principios de la RSO.

La evolución del marketing se ha concebido bajo cinco premisas: 1) la producción, 2) el producto, 3) la venta, 4) el marketing y 5) el marketing social. Actualmente, la sostenibilidad es un requerimiento para las organizaciones dadas las presiones institucionales ejercidas por las personas. Por lo tanto, su búsqueda es un requisito que permite la competitividad de la organización en el mercado [18].

En este escenario, el marketing sostenible es aquel proceso de planificación, ejecución y el control del marketing, en pro de satisfacer las necesidades de los clientes. Todo ello, mientras se es amigable con el ambiente y se encuentra alineado con los objetivos organizacionales [74]. Esta categoría tiene implícito el desarrollo sostenible. Por ende, espera conseguir como resultado final el logro de la sostenibilidad económica, ambiental y social, a través de soluciones que respondan tanto a las necesidades de los clientes como de otras partes interesadas [75].

En tal sentido, el marketing sostenible subsume a todas aquellas acepciones terminológicas del marketing que buscan, de una u otro forma, satisfacer algunos de lo que hoy se conoce como objetivos de desarrollo sostenible o que aplican algunos de los postulados de la RSO para dicho fin. Es por ello, que se considera que esta categoría es una versión completa del marketing hacia el desarrollo sostenible y se basa en la formulación de estrategias del marketing mix para satisfacer las necesidades del cliente, ámbito social; el desarrollo de procesos productivos y productos amigables con el ambiente, factor ambiental; a la vez que busca el logro de los objetivos de la organización abarcando el aspecto económico [58], [74], [76].

\section{MÉTODO}

Este documento presenta los resultados de una revisión sistémica de literatura o RSL. En general, se presentan resultados cualitativos con base en técnicas hermenéuticas para la síntesis y análisis de documentos. Se plantearon tres etapas con base en los lineamientos de la RSL [77]. Las fuentes fueron secundarias. A continuación, se describe el proceso metodológico con mayor profundidad.

\section{a. Fuentes de información}

Para identificar las fuentes de información del estudio se tuvieron en cuenta los criterios expuestos por García-Molina y Chicaíza-Becerra [78]. Todas las fuentes de información fueron secundarias. Se consultaron las bases de datos con acceso desde el Sistema de Bibliotecas de la Universidad Nacional de Colombia, SINAB, y desde el Sistema Nacional de Bibliotecas de la Corporación Universitaria Minuto de Dios. Se definió que los documentos fueran artículos científicos en revistas nacionales e internacionales, libros y capítulos de libros de presentación de resultados de investigación, informes técnicos y científicos, tesis y trabajos grado, documentos de trabajo y en menor medida documentos de ponencias y notas de prensa.

\section{b. Criterios de búsqueda}

Para ejecutar la búsqueda bibliográfica se tuvieron en cuenta varios criterios. Estos se ajustaron para cada temática. Para el caso de la responsabilidad social organizacional, se emplearon términos como: RSC, RSO, RSE y sus respectivos significados. En principio, se planteó una revisión que ocupara documentos de los diez años del periodo 2009-2019. Sin embargo, dadas las bases teóricas encontradas se decidió ampliar el criterio de búsqueda para abarcar las bases seminales acerca del tema.

Para el marketing sostenible, la búsqueda se enfocó en la identificación o diferenciación conceptual de los términos de marketing orientado a la sostenibilidad y la RSO. En tal sentido, se emplearon palabras clave como: marketing sostenible, estrategias de marketing sostenible, mix de marketing sostenible, y sus equivalencias en inglés. El periodo de búsqueda fue principalmente los últimos diez años, 2010-2020. De igual manera, se buscaron términos como: marketing ecológico, marketing verde, marketing social y marketing de perspectiva social, que fueron conceptos relacionados al tópico principal marketing sostenible.

\section{c. Análisis de la información}

Para el análisis de la información se emplearon matrices de lectura. En general, se clasificó la información en categorías según la temática. Para el caso de la RSO se utilizaron las categorías conceptuales propuestas por Tello-Castrillón y Rodríguez-Córdoba [23]. Para el marketing sostenible se categorizaron los documentos según el término que abordaran. En ese marco, se agruparon documentos con base en los conceptos claves anteriormente mencionados, con el fin de encontrar la relación entre los diferentes términos y encausar el sentido de este documento a la explicación de estrategias de marketing orientadas a la sostenibilidad en el marco de la RSO. 


\section{NECESIDAD DE SOSTENIBILIDAD Y RSO EN LAS ACCIONES DEL MARKETING}

La sostenibilidad del planeta es una necesidad de la sociedad actual. Por lo tanto, la aplicación de los lineamientos de la RSO es indispensable para que las organizaciones reduzcan su impacto en el planeta y no descarguen sus costos en la sociedad y el medio ambiente. Es decir, es indispensable que las organizaciones practiquen una RSO fundamental. Sin embargo, algunos autores podrían considerar la aplicación de la RSO o la visión hacia la sostenibilidad como un instrumento de ventaja competitiva. Esto es algo que en principio está bien, pero que podría considerarse reduccionista y desvía la verdadera intención de la RSO. En este caso se puede considerar que se aplica una RSO mecánica.

Ahora bien, existen escenarios en los que el mercado y el entorno consideran que las políticas de RSO fundamental y el enfoque de sostenibilidad aplicado por una organización son una ventaja competitiva. Al ser una apreciación externa a las decisiones de esta, no se puede considerar como una postura reduccionista. Son los grupos de interés externos los que deben identificar las acciones de RSO de la organización y no la organización la que debe alardear de ello.

La aplicación de la RSO no solo reduce el impacto de la organización sobre el ambiente, sino que garantiza la sostenibilidad empresarial y aporta satisfacción a las necesidades presentes y futuras de los consumidores a través del desarrollo sostenible. En este sentido, el marketing juega un papel fundamental, a través del desarrollo de estrategias que atiendan las nuevas tendencias y necesidades del cliente, con el fin de lograr el posicionamiento sostenido en el mercado [79].

La RSO es inherente al marketing sostenible. Por lo anterior, las organizaciones, con el fin de aportar a la sostenibilidad de la sociedad y del planeta, deben plantear estrategias que respondan a los factores económicos, ambientales y sociales.

Aplicar estrategias de marketing sostenible genera ventajas para las organizaciones. Entre estas se destaca el incremento en compromiso de los miembros frente a sus actividades. Lo anterior promueve una imagen positiva de la organización, fortalece la relación y lealtad de los consumidores y genera relaciones estables y duraderas con proveedores. Es decir, se crea valor compartido con los grupos de interés [80]-[82].

En pro de un beneficio común, se unen esfuerzos de la organización y se redefinen productos y mercado, se reorienta la cadena de valor o se desarrollan clústeres para hacer frente a una problemática social o ambiental. Todo ello paralelo a la creación de valor y se mejora la imagen corporativa [64]. Las organizaciones deben generar valor sostenible a lo largo del ciclo de vida del producto, servicio y sistema [75]. Las políticas de sostenibilidad están enmarcadas en la supervivencia de la organización, mientras se es responsable de su impacto en el entorno.

\section{ESTRATEGIAS DE MARKETING SOSTENIBLE: UNA PROPUESTA}

Con los cambios en las formas de pensar y actuar en la sociedad, se presentan nuevos escenarios que obligan a las disciplinas y campos del saber a adaptarse; el marketing no es ajeno a ello [18], [56], [62], [63], [67], [72], [75], [83]. Por lo anterior, las estrategias de marketing mix relacionadas con la adopción de prácticas sostenibles son de gran importancia. Es trascendental brindar un valor al cliente por medio de productos que respondan a sus necesidades, preferencias, y expectativas, mientras se garantiza el mínimo impacto en el ambiente y se reducen las externalidades de la organización. Paralelo a ello, al ejecutar prácticas sostenibles se garantiza el cumplimiento de los objetivos organizacionales.

El desarrollo de estrategias de marketing enfocadas a la sostenibilidad se consolida como un reto importante para las organizaciones [18], [75]. Por un lado, se deben desarrollar estudios de mercado exhaustivos para conocer las preferencias, necesidades y expectativas del cliente, y por el otro, ajustarse la oferta a las capacidades organizacionales. Con el fin de generar soluciones competitivas en mercados, todo lo anterior debe involucrar transversalmente las políticas de RSO y los grupos de interés [75]. Adicionalmente, esto genera un efecto colateral positivo. Promueve la creación relaciones redituables entre las partes.

Con base en lo anterior, a continuación, se relacionan las estrategias de marketing sostenible para cada variable del marketing mix [75], [79], [83], [84]. Para el caso del producto, las estrategias se enfocan en la alta calidad del producto a la par que se genera el menor impacto ambiental. Para ello parten del diseño y uso de materiales y embalajes ecológicos, la minimización del consumo de energía; agua y materias primas, principalmente si son recursos escasos o no renovables. De igual forma, las estrategias de marketing sostenible respecto al producto deben: a) proporcionar información completa en el empaque sobre el origen de sus componentes y su impacto ambiental; b) usar certificaciones de compromiso, por medio de etiquetas de comercio justo, producto verde, producto no testeado en animales, ecomarca, entre otras; c) no emplear pesticidas, fertilizantes inorgánicos, aditivos químicos, y otros productos similares en el proceso productivo o en el producto; y d) emplear de estándares internacionales para la gestión ambiental.

En términos de la variable precios, las estrategias se centran en i) la relación calidad precio; ii) no se establecer un precio por debajo del recomendado para incrementar ventas, ni incrementarlos excesivamente por ser un producto o servicio sostenible; iii) actividades relacionadas con la diferenciación de precios se llevan a cabo de manera honesta y ética, por ejemplo: descuentos, diversidad geográfica, precios promocionales; iv) inclusión de los costos de acciones sociales y ecológicas en el precio y v) disminución del precio ofertado mediante inversiones en equipos amigables con el medio ambiente.

Por su parte, la variable plaza está relacionada a la generación de valor en la cadena de suministro, por medio de la elección de proveedores de materias primas, productos o servicios e intermediaros que tengan participación social o ambiental entre sus actividades. Por ende, se generan relaciones de cooperación basadas en los principios de comercio ético y justo, se prioriza al proveedor local y se presta atención al impacto ambiental del proceso logístico, verbigracia, huella de carbono, embalajes, entre otros.

Por último, en la $\mathrm{P}$ de promoción, se desarrollan estrategias orientadas a generar comunicaciones claras, honestas y transparentes de marketing y publicidad. Tal es el caso de: a) desuso de descripciones incomprensibles, incompletas o engañosas; b) la creación de imagen de marca enfocada en la prosperidad social o ambiental; c) participación en actividades benéficas o de patrocinio; d) promoción del consumo sostenible; e) comunicación bidireccional con los clientes y respuesta por medio de la calidad del producto y el servicio; f) avisos periódicos a 
todos los grupos de interés sobre el compromiso del negocio con las actividades socioecológicas para generar confianza y credibilidad en la organización; g) formación periódica a los miembros de la organización en actividades sociales y medioambientales que se realizan para que puedan comunicar los beneficios de productos sostenibles para los clientes y grupos de interés; y h) reemplazo de las formas tradicionales de comunicación o publicidad por correos electrónicos, teléfono y redes sociales.

Evidentemente, las propuestas que aquí se presentan son sola una muestra del universo de posibilidades que pueden plantearse desde el marketing sostenible. El requisito indispensable es aplicar transversalmente a cada estrategia que se plantee los principios y las materias de la RSO. Con esto, además de garantizar una RSO fundamental, se garantizan políticas sostenibles. Sin embargo, esta aplicación no debe ejecutarse únicamente desde el marketing. Para que una organización sea totalmente sostenible, deberia usar los principios de la RSO en todas sus actividades de forma voluntaria y evitar, en lo posible, pensar en los beneficios económicos. Sin embargo, se entiende que esto es una dificultad para las organizaciones empresariales. Las estrategias de marketing sostenible deben plantearse y establecerse con el único fin de evitar acciones que no estén encaminadas hacia el desarrollo sostenible y la supervivencia del planeta.

Por lo anterior, la RSO direccionada a todos los aspectos de la organización podría generar una imagen positiva de la organización que le permitirá posicionarse como un referente. La tendencia hacia el consumo sostenible que tiene actualmente la sociedad [6], [85] favorece que las organizaciones que voluntariamente aplican los fundamentos de la responsabilidad social y procuran la sostenibilidad sean vistas como ejemplos a seguir y sean premiadas con el consumo de su producto.

\section{CONCLUSIONES}

Las condiciones generadas por el sistema económico hegemónico tienen repercusión en la calidad de vida de las personas. Sin embargo, aunque el panorama en algunos aspectos no es el mejor, las tendencias de cambio ofrecen un panorama prometedor. Esto, especialmente en lo referido al consumo responsable y sostenible, la recuperación de los ecosistemas, la reducción de gases de efecto invernadero emanado de las fábricas, la preocupación por las condiciones laborales justas, la seguridad de la información digital, entre otros aspectos. Para ese propósito, el marketing sostenible puede aportar un camino que permita no solo visibilizar estas prácticas, sino coaccionar a las organizaciones para la adopción de políticas de RSO fundamentales, influir en el pensamiento de los consumidores y finalmente posicionar a las organizaciones en la mente de los consumidores responsables.

A pesar de las tendencias hacia la sostenibilidad y el camino que se crea desde el marketing sostenible, aún queda mucho por trabajar desde la academia y las organizaciones. El marketing sostenible no puede ser solo un enfoque del marketing general, sino que debe convertirse en la institución cultural principal. Las personas y las organizaciones deben integrar las políticas de RSO y de sostenibilidad en todas sus acciones. En el marketing actual, el ideal debería ser el planteamiento de estrategias y la actualización de los fundamentos y postulados clásicos del marketing para que integren inherentemente las posturas de sostenibilidad y responsabilidad social que aquí se discutieron. Para ello, se proponen la realización de investigaciones que ahonden en los conceptos de ética y moral intrínsecos en el marketing; esto es un tema que se podría desarrollar a futuro.

Finalmente, hay que resaltar que todos los tipos de organizaciones, de una u otra forma, deben ejecutar un plan de mercadeo para cumplir con sus fines misionales. Por ello es importante no pensar como única consecuencia del marketing sostenible la consecución de un lucro económico a partir del valor agregado; esta es una consecuencia positiva para las organizaciones empresariales. Las organizaciones sin fines de lucro como hospitales, universidades, fundaciones, entre otras, también están en la obligación de aplicar lo planteado en este documento.

\section{REFERENCIAS}

[1] B. Ramírez, “Globalización o imperio: ¿nuevas tendencias del capitalismo contemporáneo?”, ACME: An International Journal for Critical Geographies, vol. 2, núm. 2, pp. 236-241, 2003.

[2] J. de Rivera, Á. J. Gordo-López, y P. Cassidy, "La economía colaborativa en la era del capitalismo digital”, Revista de Estudios para el Desarrollo Social de la Comunicación, núm. 15, pp. 20-31, 2017, doi: 10.15213/redes.n15.p22.

[3] Naciones Unidas, “Agenda 2030 y los Objetivos de Desarrollo Sostenible. Una oportunidad para América Latina y el Caribe”. Publicación de las Naciones Unidas, ene-2018 [Online]. Disponible en: https://goo.gl/DD6dR4

[4] The Global Compact, "Los diez principios del Pacto Global". Naciones Unidas, 26-jul-2000 [Online]. Disponible en: http://www.pactoglobal.uy/index.php/pacto-global/10-principios. [Consultado: 10-mar-2016]

[5] Comisión de las Comunidades Europeas, "Libro Verde. Fomentar un marco europeo para la responsabilidad social de las empresas". Comisión Europea, $2001 \quad$ [Online]. http://www.europarl.europa.eu/meetdocs/committees/deve/20020122/com(2001)366_es.pdf. [Consultado: 25-jun-2016]

[6] C. Camacho-Delgado, "Del consumismo al consumo sostenible", Punto de Vista, vol. 4, núm. 6, pp. 111-124, 2013, doi: 10.15765/pdv.v4i6.405.

[7] H. Katrandjiev, "Ecological marketing, green marketing, sustainable marketing: Synonyms or an evolution of ideas?", Economic Alternatives, 1, pp. 71-82, 2016.

[8] C. E. López-Rodríguez y L. A. Arévalo, "Del marketing ecológico al greenwashing: una mirada en escenarios comerciales colombianos e internacionales”, Ciencias Económicas, vol. 16, núm. 01, pp. 9-37, 2019.

[9] K. B. Kumar, R. G. Rajan, y L. Zingales, “What determines firm size?”, National Bureau of Economic Research, Cambridge, documento de trabajo 7208, jul. 1999 [Online]. Disponible en: http://www.nber.org/papers/w7208. [Consultado: 15-abr-2020]

[10] Z. Bauman, "Trabajo, consumismo y nuevos pobres", España: Gedisa, 2000.

[11] Z. Bauman, "Vida de consumo", México: Fondo de Cultura Económica, 2007.

[12] C. Hernández-Garnica y C. A. Maubert Viveros, "Fundamentos de marketing", en Fundamentos de marketing, 2.a ed., México: Pearson Educación, 2017, pp. 3-34.

[13] P. Kotler y G. Armstrong, "Fundamentos de marketing", 10.a ed. México: Pearson Educación, 2013. 
[14] R. M. Marini, "Proceso y tendencias de la globalización capitalista", en La teoría social latinoamericana: cuestiones contemporáneas, México: Ediciones El Caballito, 1994, pp. 49-68.

[15] G. Armstrong y P. Kotler, Marketing, 11.a ed. México: Pearson Education, 2012.

[16] J. McCarthy y W. D. Perreault, Basic Marketing. A Global Managerial Approach, 11.a ed. Boston, MA: Irwin, 1993

[17] American Marketing Association AMA, "The definition of marketing", American Marketing Association. [Online]. Disponible en: https://www.ama.org/the-definition-of-marketing-what-is-marketing/. [Consultado: 11-oct-2020].

[18] V. Kumar, Z. K. Rahman, A. A. Kazmi, y P. Goyal, "Evolution of sustainability as marketing strategy: beginning of new era", Procedia Social and Behavioral Sciences, vol. 37, pp. 482-489, ene. 2012, doi: 10.1016/j.sbspro.2012.03.313

[19] W. D. Perreault, J. P. Cannon, y J. McCarthy, Essentials of Marketing, 13.a ed. EE.UU: McGraw-Hill, 2011.

[20] R. A. Kerin, S. W. Hartley, y W. Rudelius, Marketing, 11.a ed., México: McGraw-Hill, 2014.

[21] W. J. Stanton, M. J. Etzel, y B. J. Walker, "Fundamentos de marketing”, 14.a ed., México: Mc Graw Hill, 2007.

[22] J. Ríos Szalay, “La estandarización terminológica de la administración, un empeño amplio y persistente, ¿pero inviable?”, presentado en XV Congreso Internacional de Contaduría, Administración e Informática, México, 2010, pp. 1-37.

[23] C. Tello-Castrillón y M. P. Rodríguez-Córdoba, "Categorías conceptuales para el estudio de la responsabilidad social organizacional", Hallazgos, vol. 11, núm. 22, pp. 119-135, 2014, doi: 10.15332/s1794-3841.2014.0022.07.

[24] C. Tello-Castrillón y M. P. Rodríguez-Córdoba, "Fundamentos ético disciplinares de la responsabilidad social organizacional”, Revista Venezolana de Gerencia, vol. 21, núm. 75, pp. 491-508, 2016.

[25] C. Tello-Castrillón y J. Londoño-Cardozo, "Responsabilidad Social Organizacional, definiciones y aplicación", en La construcción organizacional de la responsabilidad social: fundamentos teóricos y casos de estudio, Palmira, Valle del Cauca: Universidad Nacional de Colombia, 2020, pp. 75-113.

[26] C. Tello-Castrillón, "Relación entre el gobierno corporativo y la responsabilidad social organizacional: estudio de caso en una empresa multilatina colombiana, Carvajal, S.A.", Tesis doctoral, Universidad Nacional de Colombia sede Manizales, Manizales, Colombia, 2018 [Online]. Disponible en: http://bdigital.unal.edu.co/65743/1/16774542.2018.pdf. [Consultado: 11-oct-2020].

[27] C. Dávila, "Teorías organizacionales y administración. Enfoque crítico", 2.a ed., Bogotá, Colombia: Editorial Presencia LTDA, 2001.

[28] C. Tello-Castrillón, "El concepto de organización, tan cerca y tan lejos", en Conjeturas organizacionales: fundamentos para el estudio de la organización, Bogotá: Editorial Universidad Nacional de Colombia, 2018, pp. 79-102.

[29] D. C. North, "Instituciones, cambio institucional y desempeño económico", 3.a reimpresión. México: Fondo de cultura Económica, 2006.

[30] D. C. North, "Instituciones, ideología y desempeño económico". Elcato.org, 2003, ene. 24. [Online]. Disponible en: https://www.elcato.org/instituciones-ideologia-y-desempeno-economico

[31] C. Castoriadis, La institución imaginaria de la sociedad, México: Tusquets Editores, 2013.

[32] W. Gonzáles, "Humano posthumano en la antropobiología contemporánea", presentado en Seminario internacional humanismo y posthumanismo en la postmodernidad: su impacto en la administración pública y la gobernanza, Cali, 2018, pp. 65-81.

[33] M. T. Méndez-Picazo, "Ética y responsabilidad social corporativa", Información Comercial Española, ICE: Revista de Economía, núm. 823 , pp. 141-151, 2005.

[34] J. Londoño-Cardozo y I. Hernández-Madroñero, "El problema de los conceptos científicos en la clasificación de las organizaciones", en Conjeturas organizacionales. Fundamentos para el estudio de la organización, Bogotá: Editorial Universidad Nacional de Colombia, 2018, pp. 103-128.

[35] C. Tello-Castrillón, "Responsabilidad social organizacional, Estado colombiano y post-acuerdo", en Derechos laborales, fomento económico, informalidad y desarrollo, Bogotá, Colombia: Senado de la República - Centro de Altos Estudios Legislativos, 2018, pp. 152171.

[36] N. Gorrochategui, V. Martins- De Oliveira, J. Domínguez-Granda, y H. Chero Valdivieso, "Responsabilidad social de las organizaciones: avances y propuestas en América Latina”, Chimbote, Perú: Universidad Católica Los Ángeles de Chimbote, 2015.

[37] N. Gorrochategui, V. Martins de Oliveira, y N. Stevenson Palamara, "Responsabilidad social de las organizaciones (RSO): estudios desde la mirada de la responsabilidad social hacia los Objetivos de Desarrollo Sostenible en América Latina", Santiago de Chile: Universidad Autónoma de Chile, 2019.

[38] M. Friedman, "La responsabilidad social de la empresa es incrementar sus beneficios", The New York Times Magazine, vol. 1, pp. 122$126,1970$.

[39] M. S. Aßländer, "Corporate social responsibility as subsidiary co-responsibility: A macroeconomic perspective", Journal of Business Ethics, vol. 99, núm. 1, pp. 115-128, 2011, doi: 0.1007/s10551-011-0744-x.

[40] G. Enderle, "Clarifying the terms of business ethics and CSR", Business Ethics Quarterly, vol. 20, núm. 4, pp. 730-732, 2010.

[41] B. Ungericht y C. Hirt, "CSR as a political arena: the struggle for a European framework", Business and Politics, vol. 12, núm. 4, pp. 122, 2010.

[42] C. M. Perrone, S. Engelman, y A. R. Reppold-Filho, "Desafíos contemporáneos de la Ética y de la Moral en las organizaciones", Invenio: Revista de Investigación Académica, vol. 17, núm. 31-32, pp. 33-47, 2014.

[43] R. Jenkins, "What is corporate social responsibility?", en Handbook of Economics and Ethics, Cheltenham (UK): Edward Elgar Publishing Limited, 2009, pp. 69-76.

[44] L. J. Lozano-Correa, "La confianza y su incidencia en los grupos de interés", en Responsabilidad social de las organizaciones (RSO): aportes teórico-prácticos para lograr los Objetivos de Desarrollo Sostenible en América Latina, Bogotá, Colombia: Universidad Santo Tomás, 2017, pp. 481-492.

[45] E. Olivero-Arias, R. Morales-Vergara, y M. Alvarado-Márquez, "Identificación de los principios de los stakeholders en una asociación de agricultores arroceros, componente del desarrollo rural", en Responsabilidad social de las organizaciones (RSO): Aportes TeóricoPrácticos para lograr los Objetivos de Desarrollo Sostenible en América Latina, Bogotá, Colombia: Universidad Santo Tomás, 2017, pp. $147-172$

[46] J. R. Volpentesta, "Procedimientos administrativos en la gestión de los stakeholders", en Responsabilidad Social de las Organizaciones: avances y propuestas en América Latina, Chimbote, Perú: Universidad Católica Los Ángeles de Chimbote, 2015, pp. 59-90.

[47] J. R. Volpentesta, "Método de integración de stakeholders a procesos de toma de decisiones de las empresas", en Responsabilidad social de las organizaciones (RSO): Aportes teórico-prácticos para lograr los Objetivos de Desarrollo Sostenible en América Latina, Bogotá, Colombia: Universidad Santo Tomás, 2017, pp. 89-108.

[48] C. Tello-Castrillón, M. del P. Rodríguez-Córdoba, y E. Varela-Barrios, "El gobierno corporativo de las multilatinas y su responsabilidad social organizacional”, presentado en XVIII Congreso Internacional de Contaduría, Administración e Informática, México, 2013 [Online]. 
3TDLEL\%20GOBIERNO\%20CORPORATIVO\%20DE\%20LAS.pdf

[49] L. S. Acosta, J. Londoño-Cardozo, y B. Gaitán-Vera, "La economía colaborativa como instrumento que transforma al hombre en un ser socialmente responsable", en La construcción organizacional de la responsabilidad social: fundamentos teóricos y casos de estudio, Palmira, Valle del Cauca: Universidad Nacional de Colombia, 2020, pp. 143-167.

[50] ISO, "ISO 26000:2010 Guía de responsabilidad social", 2010. [Online]. Disponible en: https://www.iso.org/obp/ui\#iso:std:iso:26000:ed1:v1:es. [Consultado: 11-jul-2017]

[51] C. Tello-Castrillón, "Neoinstitucionalismo, responsabilidad social organizacional y gobernanza", Cuestiones Políticas, vol. 30, núm. 52, pp. 116-130, 2014.

[52] R. E. Sosa-Cardona, C. Tello-Castrillón, y E. F. Pineda-Henao, "Derecho constitucional, responsabilidad social organizacional y gobernanza: el caso de empresas municipales de Cali”, Justicia, vol. 25, núm. 37, pp. 227-244, jun. 2020, doi: 10.17081/just.25.37.4153.

[53] J. Elkington, "Accounting for the triple bottom line", Measuring Business Excellence, vol. 2, núm. 3, pp. 18-22, 1998, doi: $10.1108 / \mathrm{eb} 025539$.

[54] J. Elkington, "Enter the triple bottom line", en The Triple Bottom Line: Does It All Add Up, Wales, Reino Unido: Taylor \& Francis, 2004, pp. 1-16.

[55] J. Londoño-Cardozo, "Propuesta de caracterización de la responsabilidad digital organizacional de la economía colaborativa en Colombia", Tesis inédita de pregrado, Universidad Nacional de Colombia, Palmira, 2020.

[56] T. A. Monteiro, A. C. Giuliani, J. Cavazos-Arroyo, y N. K. Pizzinatto, "Mezcla del marketing verde: una perspectiva teórica", Cuadernos del CIMBAGE, núm. 17, p. 103-126, 2015.

[57] Naciones Unidas, "Asamblea General de las Naciones Unidas". [Online]. Disponible en: https://www.un.org/es/ga/president/65/issues/sustdev.shtml. [Consultado: 14-oct-2020]

[58] C. L. Giraldo Patiño, D. C. Micolta Rivas, y E. Oneill Marmolejo, "Revisión de literatura sobre el marketing sostenible y sus relaciones conceptuales", presentado en I Congreso internacional en ciencias económicas y especiales. II Encuentro de socialización académica sobre experiencias investigativas, Cali, 2020.

[59] D. Maftei, "Ecomarketing for the company? Why?", The USV Annals of Economics and Public Administration, vol. 12, núm. 2(16), pp. 106-112, dic. 2013.

[60] H. Salas-Canales, "Marketing ecológico y comportamiento proambiental en estudiantes de un instituto privado de Lima (Perú), 2019", Ciencia Unemi, vol. 13, núm. 34, pp. 94-104, sep. 2020.

[61] J. V. Calomarde, "Marketing Ecológico", presentado en Marketing Ecológico. Madrid: Pirámide, 2005, p. 16 [Online]. Disponible en: http://www.relec.es/archivos/sostenibilidad/Jose_V_Calomarde_W.pdf. [Consultado: 15-oct-2020].

[62] F. M. Belz, K. Peattie, y J. M. Gali, "Marketing de sostenibilidad”. Profit Editorial, 2013.

[63] M. J. Polonsky, “An Introduction to Green Marketing”, EGJ, vol. 1, núm. 2, nov. 1994, doi: 10.5070/G31210177. [Online]. Disponible en: https://escholarship.org/uc/item/49n325b7. [Consultado: 10-oct-2020]

[64] A. E. Aguilar, "Marketing verde, una oportunidad para el cambio organizacional”, Realidad y Reflexión, vol. 16, núm. 44, pp. 92-106, 2016, doi: 10.5377/ryr.v44i0.3567.

[65] V. N. Lopes y M. N. Pacagnan, "Marketing verde e práticas socioambientais nas indústrias do Paraná”, RAUSP, vol. 49, núm. 1, pp. 116128, 2014, doi: 10.5700/rausp1135.

[66] J. M. Mendoza-Guerra, "Epistemología de la administración: objeto, estatuto, desarrollo disciplinar y método", Revista científica Pensamiento y Gestión, núm. 45, pp. 211-238, 2018, doi: 10.14482/pege.41.9704.

[67] K. Peattie y S. Peattie, "Social marketing: A pathway to consumption reduction?", Journal of Business Research, vol. 62, núm. 2, pp. 260268, feb. 2009, doi: 10.1016/j.jbusres.2008.01.033.

[68] P. Kotler, "Dirección de Marketing", 15.a ed. México: Pearson Educación de México S.A de C.V., 2016 [Online]. Disponible en: http://www.ebooks7-24.com.ezproxy.uniminuto.edu/stage.aspx?il=\&pg=\&ed=273. [Consultado: 15-oct-2020].

[69] G. Guerrero-Pino, "Introducción a la Filosofía de la Ciencia” Documentos de Trabajo, 4.a. Santiago de Cali: Universidad del Valle, 2015.

[70] J. M. Mardones, "Filosofía de las ciencias humanas y sociales: nota histórica de una polémica incesante", filosofía de las ciencias humanas y sociales: materiales para una fundamentación científica, vol. 1, Barcelona: Anthropos Editorial, 1991, pp. 19-57.

[71] E. F. Pineda-Henao, "Filosofía de la ciencia aplicada a la administración", Revista Ensayos, vol. 5, núm. 5, pp. 177-192, 2012.

[72] M. C. Bustillo-Castillejo, "El marketing social una respuesta del mundo mercantil al desarrollo sostenible", Saber, ciencia y libertad, vol. 6, núm. 2, pp. 169-176, jul. 2011, doi: 10.18041/2382-3240/saber.2011v6n2.2504.

[73] J. Elkington, Cannibals with Forks: The Triple Bottom Line of 21st Century Business, Oxford: Capstone Publishing Ltd., 1997.

[74] D. Fuller, "Marketing Mix Design-for-Environment (Dfe): A Systems Approach", Journal of Business Administration and Policy Analysis, p. 309, ene. 1999.

[75] M. Charter, K. Peattie, J. Ottman, y M. J. Polonsky, "Marketing and sustainability. Centre for Business Relationships, Accountability, Sustainability and Society (BRASS)", Centre for Sustainable Design, abr-2002 [Online]. Disponible en: https://cfsd.org.uk/smart-knownet/smart-know-net.pdf. [Consultado: 15-oct-2020].

[76] E. G. Iracheta y S. U. Enales, "Marketing sostenible y responsabilidad social corporativa: activos efectivos y de gran competitividad", Tesis de grado, Universidad Euskal Herriko del País Vasco Unibertsitatea, Bilbao, 2016 [Online]. Disponible en: https://addi.ehu.es/bitstream/handle/10810/18976/EduardoGonzalezIracheta_TFG.pdf?sequence=2\&isAllowed=y. [Consultado: 15-oct2020].

[77] L. A. Chicaíza-Becerra, M. I. Riaño-Casallas, S. P. Rojas-Berrío, y C. Garzón-Santos, "Revisión sistemática de literatura en Administración”. Facultad de Ciencias Económicas. Centro de Investigaciones para el Desarrollo - CID, jun-2017 [Online]. Disponible en: $\quad$ http://www.fce.unal.edu.co/centro-editorial/documentos/escuela-de-administracion-y-contaduria-publica/1600-29-revisionsistematica-de-la-literatura-en-administracion.html. [Consultado: 15-oct-2020].

[78] M. García-Molina y L. Chicaíza-Becerra, "Guía de fuentes para la investigación en Ciencias Económicas". Facultad de Ciencias Económicas. Centro de Investigaciones para el Desarrollo - CID, 21-feb-2011. Doi http://dx.doi.org/10.2139/ssrn.1766062

[79] E. Y. Mendoza Vargas, J. A. Boza Valle, H. E. Escobar Terán, y G. H. Macías España, "El marketing verde como generador de valor superior en los emprendimientos", Revista Órbita Pedagógica, vol. 4, núm. 2, pp. 129-144, 2017.

[80] M. E. Porter y M. R. Kramer, "La creación de valor compartido", Harvard Business Review, pp. 1-18, 2011.

[81] M. E. Porter y M. R. Kramer, "The competitive advantage of corporate philanthropy", Harvard Business Review, vol. 80, núm. 12, pp. $56-68,2002$. 
[82] M. E. Porter y M. R. Kramer, "Strategy and society: The link between competitive advantage and corporate social responsibility", Harvard Business Review, vol. 84, núm. 12, pp. 77-92, 2006.

[83] E. Rudawska, "Sustainable marketing strategy in food and drink industry: a comparative analysis of B2B and B2C SMEs operating in Europe | Emerald Insight”, Journal of Business \& Industrial Marketing, vol. 34, núm. 4, pp. 875-890, jun. 2019, doi: https://doi.org/10.1108/JBIM-05-2018-0171.

[84] M. Martínez-Merino, "Marketing sostenible: estrategias y percepción del consumidor vasco", Tesis de grado, Universidad del País Vasco, Bilbao, 2020 [Online]. Disponible en: https://addi.ehu.es/bitstream/handle/10810/47022/TFG_Maria\%20Martinez.pdf?sequence=3\&isAllowed=y. [Consultado: 08-nov-2020]

[85] E. Ortiz de Mendivil-Llano, "El consumo sostenible como perspectiva innovadora", Tesis de maestría, Universidad Internacional de Andalucía, Sevilla, España, 2010 [Online]. Disponible en: http://biblioteca.utec.edu.sv/siab/virtual/elibros_internet/55728.pdf. 